\section{Balloon-Assisted Cannulation for Difficult Anterior Cerebral Artery Access}

\author{
Varun Naragum ${ }^{a} \quad$ Mohamad AbdalKader $^{b}$ Thanh N. Nguyen ${ }^{a, c}$ \\ Alexander Norbash ${ }^{d}$ \\ Departments of a Neurology and Radiology, ${ }^{\mathrm{b}}$ Radiology, and ${ }^{\mathrm{C}}$ Neurology, Neurosurgery \\ Radiology, Boston Medical Center, Boston, MA, and d Department of Radiology, University \\ of California, San Diego, CA, USA
}

\section{Keywords}

Anterior cerebral artery complex · Cerebral aneurysm · Aneurysm coiling · Balloon inflation

\section{Abstract}

The anterior communicating artery is a common location for intracranial aneurysms. Compared to surgical clipping, endovascular coiling has been shown to improve outcomes for patients with ruptured aneurysms and we have seen a paradigm shift favoring this technique for treating aneurysms. Access to the anterior cerebral artery can be challenging, especially in patients with tortuous anatomy or subarachnoid hemorrhage or in patients presenting with vasospasm. We present a technique for cannulating the anterior cerebral artery using a balloon inflated in the proximal middle cerebral artery as a rebound surface.

(C) 2017 S. Karger AG, Basel

\section{Introduction}

The incidence of intracranial aneurysms in the population is estimated to be $3.2 \%$ [1]. Approximately $30-40 \%$ of intracranial aneurysms occur in relation to the anterior cerebral artery (ACA). The anterior communicating artery complex is a common location for ruptured aneurysms [2]. Distal ACA aneurysms form a particularly rare and difficult-to-treat subgroup of ACA aneurysms. Endovascular coiling has been shown to improve functional outcome when compared with surgical clipping following aneurysmal subarachnoid hemorrhage [3]. 

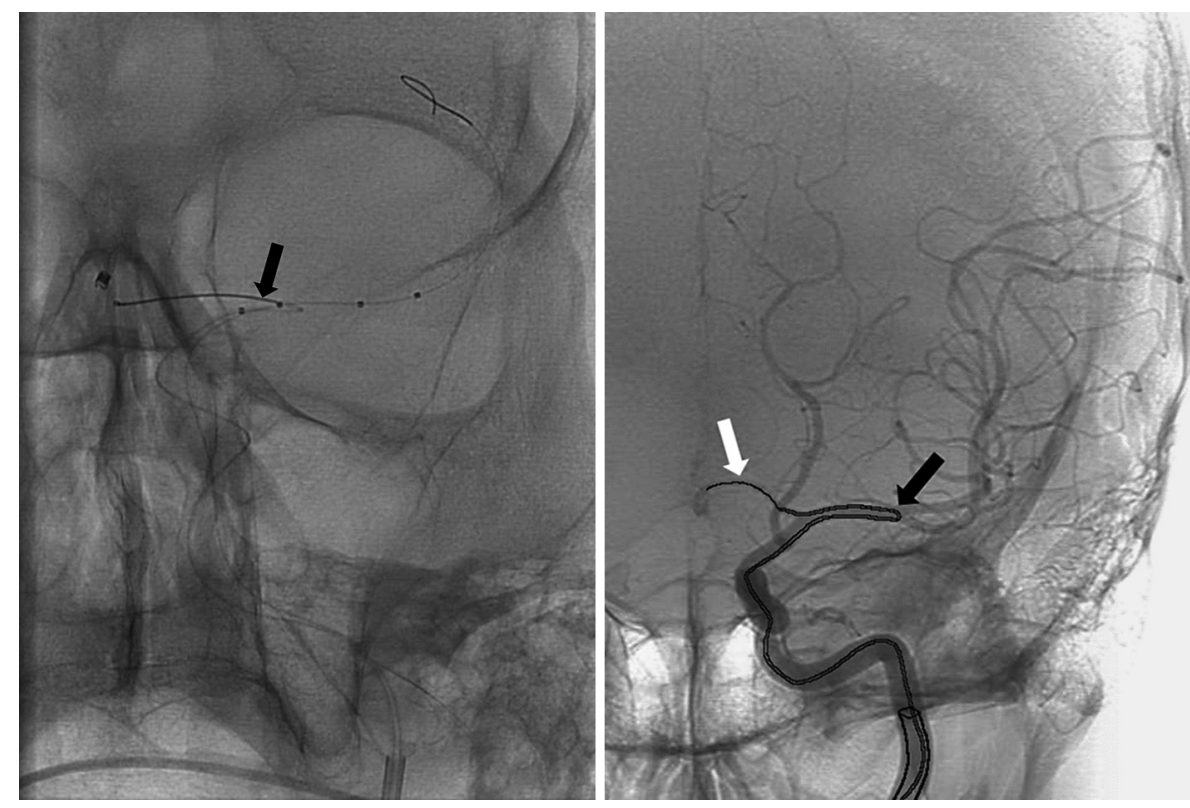

Fig. 1. Embolization microcatheter herniating within the ipsilateral middle cerebral artery (black arrow). Microwire in the anterior cerebral artery (white arrow). The image on the left represents our case. The image on the right is an illustration.

Several studies have shown that endovascular coiling of unruptured aneurysms is associated with low comparative perioperative morbidity and mortality [4-7]. With the evolution in treatment of aneurysms, improvements in vascular access and embolization techniques are facilitating, permitting expansion of patient eligibility for endovascular treatment [8]. Occasionally, access to the ACA and procedural microcatheter stability within the aneurysm can be challenging because of the acute angulation of the A1 segment origin at its origin from the larger-diameter carotid terminus [9]. Presence of vasospasm may accentuate the challenge of cannulating the small diameter of the ACA. Attempts to cannulate the ACA complex can be associated with herniation of the microwire, microcatheter, or both into the ipsilateral middle cerebral artery (MCA) (Fig. 1). Under such circumstances, the microcatheter tip may fail to advance within the ACA or fail to achieve stable positioning within the vessel allowing safe and effective treatment. Repeated attempts to cannulate the ACA can lead to prolonged procedure times and potentially to an increased risk of instrumentation complications such as dissection and stroke.

To overcome these concerns, we propose a technique to help guide the microcatheter and microwire into the ACA. A compliant balloon (either Hyperform balloon or Scepter balloon) can be navigated into the proximal ipsilateral MCA. The embolization microcatheter is advanced close to the ACA origin. Intravenous heparin is administered to keep the activated clotting time (ACT) above 250 seconds. The balloon is transiently inflated in the proximal MCA (Fig. 2) to act as a barricade and rebound surface preventing herniation of the embolization microcatheter into the MCA and encouraging facilitated cannulation of the ACA. The embolization microcatheter is advanced over a microwire into the ACA. Once satisfactory positioning of the microcatheter in the aneurysm is achieved, the balloon is deflated. Reinflation of the balloon is performed during coiling of aneurysm if further balloon remodeling or hemostasis prophylaxis is required. 

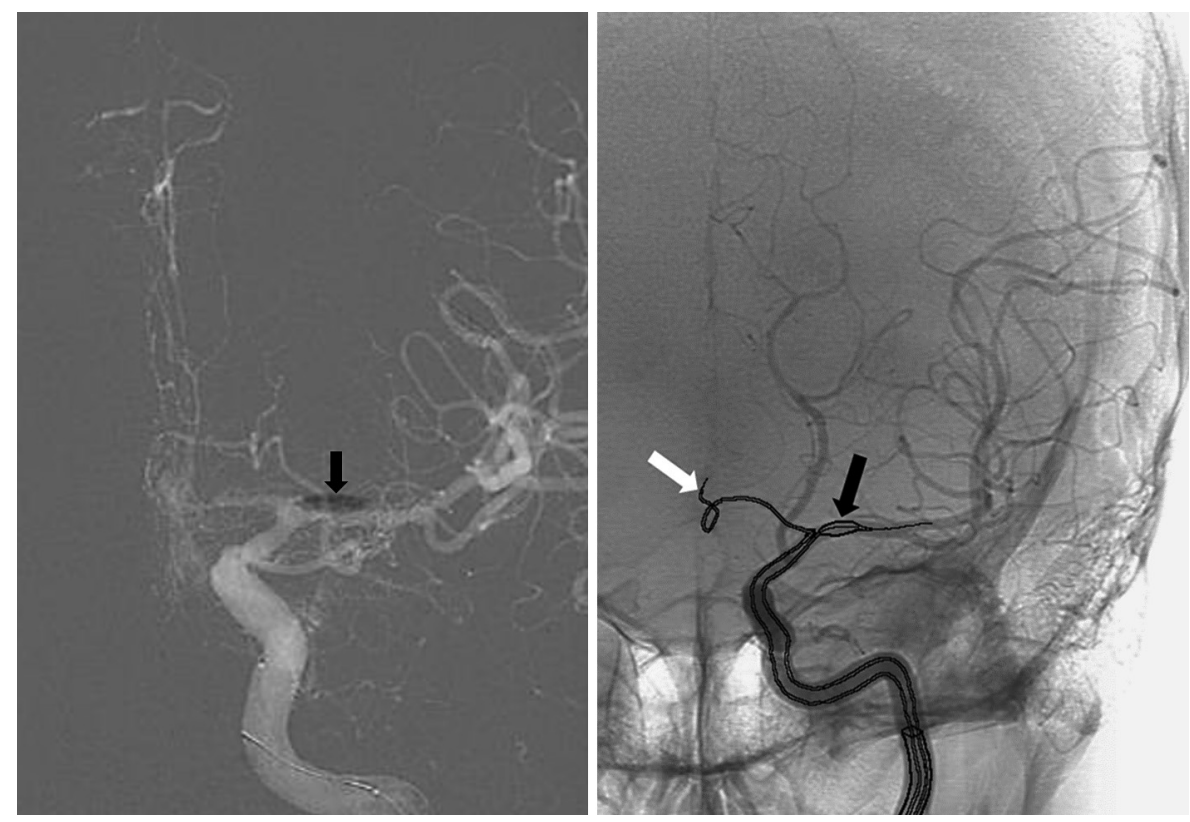

Fig. 2. Balloon inflated in the proximal middle cerebral artery (black arrow) and embolization microcatheter successfully advanced into the anterior cerebral artery (white arrow). The image on the left is our case. The image on the right is an illustration.

\section{Case Report}

A 62-year-old man complained of headaches a week prior to presentation. He had a fluctuating level of arousal over the week, and was found down on the day of admission. Head CT showed subarachnoid hemorrhage. CT angiogram displayed a 3-mm anterior communicating artery aneurysm and diffuse vasospasm. Based on the history and imaging findings it was felt that the true ictus was 7 days prior to admission. The patient was taken to the angiography suite for intervention. The entire procedure was performed under general anesthesia, with invasive arterial pressure monitoring to assist us in maintaining accurate hemodynamic parameters. A 6-French 11-cm Pinnacle femoral access sheath (Terumo, Somerset, NJ, USA) was used for groin access. A 6-French Envoy MPD guide catheter (Cordis Neurovascular, Miami Lakes, FL, USA) was placed in the proximal left cervical internal carotid artery. Carotid angiogram confirmed the presence of a 3-mm anterior communicating artery aneurysm. In addition, severe vasospasm of the left ACA A2 segment was also seen. Attempts to cannulate the left ACA were undertaken via the left carotid artery with an SL-10 microcatheter (Boston Scientific, Natick, MA, USA), utilizing a Synchro 014 microwire (Boston Scientific, Fremont, CA, USA). The efforts were initially unsuccessful using several microwires, in part due to vasospasm of the ACA and the consequential narrowed luminal vascular diameter of the ACA. Vasodilator drugs were not given before embolization of the aneurysm. Attempts to cannulate the aneurysm resulted in repeated prolapse and loop formation of the microcatheter in the left MCA. 2,000 units of intravenous heparin were given before placement of the microcatheter in the supraclinoid internal carotid artery. An ACT goal close to 200 seconds was targeted. A Scepter XC balloon (Microvention, Tustin, CA, USA) over a Synchro 014 microwire (Boston Scientific, Fremont, CA, USA) was navigated to the proximal left MCA and briefly inflated at the M1 stem, for no more than 2 minutes. The patient's hemodynamic parameters were monitored during this time by the anesthesia team. This enabled deflection of the SL-10 microcatheter into the ACA and subsequently the anterior communicating artery aneurysm. The balloon was deflated after successful cannulation of the aneurysm. The balloon was then navigated across the aneurysm, re-inflated for less than 2 minutes, during placement of the Deltapaq coil (Micrus Endovascular, San Jose, CA, USA) inside the aneurysm. Satisfactory occlusion of the aneurysm was achieved. Intra-arterial Verapamil was then given to treat vasospasm. The patient was monitored in the neurological intensive care unit over the following weeks. He made a full neurological recovery and was discharged home. At 6 months' follow-up, he remained neurologically intact. 


\section{Discussion}

This technical case report illustrates the use of a balloon in the ipsilateral M1 segment to help guide the microcatheter into the ACA in cases of difficult A1 segment access due to arterial vasospasm or vessel tortuosity. Other techniques that may be used to help achieve cannulation stability of the A1 segment are (1) placement of the microwire high in the ipsilateral ACA prior to attempted microcatheter tracking over the microwire, (2) use of an intermediate catheter for stability as a triaxial approach, or (3) higher placement of the guide catheter than is conventionally undertaken. In our described instance, the attempt to place the microwire high into the ipsilateral A2 was precluded by repetitive wire herniation into the ipsilateral M1 segment. We already had a prepared microballoon due to our intention to use a safety balloon for hemostatic prophylaxis protection in the event of procedure rupture [10], particularly given the increased rupture rate of smaller aneurysms such as that treated in this instance. Navigation of the balloon to the ipsilateral MCA was in our estimation a logical step rather than removing the whole system and placing an intermediate catheter as part of a triaxial system, or changing to a guide catheter that could permit more distal placement.

In addition to the technique we describe in this article, we have routinely utilized the balloon remodeling technique with temporary inflation of a nondetachable balloon for embolization of difficult-to-treat aneurysms. The balloon remodeling technique has been shown to achieve better occlusion of wide-necked aneurysms, without increasing the risk of the procedure $[11,12]$. This technique has also been shown to achieve better intermediate-term angiographic and clinical outcomes, with acceptable morbidity and mortality rates [13]. We have also used balloon guide catheters to achieve flow arrest during mechanical thrombectomy for acute stroke [14] as well as during carotid stent placement.

In instances such as described in our report we believe two precautionary considerations are in order. First, we are mindful of the need, particularly when occluding intracranial flow even briefly, to be meticulous where the patient's anticoagulation status is concerned, and to adjust the ACT to minimize any risk of thromboemboli. Second, we are aware of the risk of redirecting additional blood flow from the MCA to the weakened ACA harboring the target aneurysm as a direct result of the temporary balloon occlusion. We therefore suggest close attention to the patient's hemodynamic status, with the aid of invasive arterial pressure monitoring, during such maneuvers with pressure control as necessary, to minimize the risk of intraprocedural aneurysm rupture. At our institution, we have not used any form of intraoperative neurophysiological monitoring for our cases. Neurophysiological monitoring utilizing electroencephalography, somatosensory evoked potentials, or brainstem auditory evoked potentials may be a valuable adjunct to neurointerventional procedures [15].

In conclusion, in cases with difficult access to the ACA, balloon inflation in the ipsilateral MCA can be performed to deflect the microcatheter into the A1 segment.

\section{Statement of Ethics}

Informed consent was obtained. No human study or animal experiment regulations were violated.

\section{Disclosure Statement}

The authors have no conflicts of interest to declare. 


\section{References}

1 Thompson BG, Brown RD, Amin-Hanjani S, Broderick JP, Cockroft KM, Connolly ES, Duckwiler GR, Harris CC, Howard VJ, Johnston SC, Meyers PM: Guidelines for the management of patients with unruptured intracranial aneurysms. Stroke 2015;46:2368-2400.

2 Kassell NF, Torner JC, Haley Jr EC, Jane JA, Adams HP, Kongable GL: The International Cooperative Study on the Timing of Aneurysm Surgery: Part 1: Overall management results. J Neurosurg 1990;73:18-36.

3 Molyneux A, et al; International Subarachnoid Aneurysm Trial (ISAT) Collaborative Group: International Subarachnoid Aneurysm Trial (ISAT) of neurosurgical clipping versus endovascular coiling in 2143 patients with ruptured intracranial aneurysms: a randomised trial. Lancet 2002;360: 1267-1274.

4 Alshekhlee A, Mehta S, Edgell RC, Vora N, Feen E, Mohammadi A, Kale SP, Cruz-Flores S: Hospital mortality and complications of electively clipped or coiled unruptured intracranial aneurysm. Stroke 2010;41:1471-1476.

5 Brinjikji W, Rabinstein AA, Nasr DM, Lanzino G, Kallmes DF, Cloft HJ: Better outcomes with treatment by coiling relative to clipping of unruptured intracranial aneurysms in the United States, 2001-2008. Am J Neuroradiol 2011;32:1071-1075.

6 Barker FG, Amin-Hanjani S, Butler WE, Hoh BL, Rabinov JD, Pryor JC, Ogilvy CS, Carter BS: Age-dependent differences in short-term outcome after surgical or endovascular treatment of unruptured intracranial aneurysms in the United States, 1996-2000. Neurosurgery 2004;54:18-30.

7 McDonald JS, McDonald RJ, Fan J, Kallmes DF, Lanzino G, Cloft HJ: Comparative effectiveness of unruptured cerebral aneurysm therapies. Stroke 2013;44:988-994.

8 Lin N, Cahill KS, Frerichs KU, Friedlander RM, Claus EB: Treatment of ruptured and unruptured cerebral aneurysms in the USA: a paradigm shift. J Neurointerv Surg 2012;4:182-189.

9 Graves VB: Advancing loop technique for endovascular access to the anterior cerebral artery. Am J Neuroradiol 1998;19:778-780.

10 Nguyen TN, Raymond J, Guilbert F, Roy D, Mahmoud M, Berube M, Weill A: Association of endovascular therapy of very small ruptured aneurysms with higher rates of procedure-related rupture. J Neurosurg 2008;108: 1088-1092.

11 Moret J, Cognard C, Weill A, Castaings L, Rey A: Reconstruction technic in the treatment of wide-neck intracranial aneurysms. Long-term angiographic and clinical results. Apropos of 56 cases. J Neuroradiol 1997;24: 30-44.

12 Pierot L, Cognard C, Spelle L, Moret J: Safety and efficacy of balloon remodeling technique during endovascular treatment of intracranial aneurysms: critical review of the literature. Am J Neuroradiol 2012;33:12-15.

13 Malek AM, Halbach VV, Phatouros CC, Lempert TE, Meyers PM, Dowd CF, Higashida RT: Balloon-assist technique for endovascular coil embolization of geometrically difficult intracranial aneurysms. Neurosurgery 2000;46:1397-1407.

14 Nguyen TN, Malisch T, Castonguay AC, Gupta R, Sun CH, Martin CO, Holloway WE, Mueller-Kronast N, English JD, Linfante I, Dabus G: Balloon guide catheter improves revascularization and clinical outcomes with the Solitaire device. Stroke 2014;45:141-145.

15 Liu AY, Lopez JR, Do HM, Steinberg GK, Cockroft K, Marks MP: Neurophysiological monitoring in the endovascular therapy of aneurysms. Am J Neuroradiol 2003;24:1520-1527. 